REVIEW ARTICLE

\title{
An Alternative Strategy to Manage a Large Perforation of the Schnei- derian Membrane by Staged Re-entry into the Maxillary Sinus: Ob- served Clinical and Histological Changes of the Regenerated Schnei- derian Membrane
}

\author{
Cameron YS Lee $e^{1,2 *}$ \\ ${ }^{1}$ Private Practice in Oral, Maxillofacial and Reconstructive Surgery, USA \\ ${ }^{2}$ Department of Periodontology and Oral Implantology, Temple University Kornberg School of Dentistry, Philadelphia, USA
}

*Corresponding author: Cameron YS Lee, Adjunct Professor of Surgery, Department of Periodontology and Oral Implantology, Temple University Kornberg School of Dentistry, Philadelphia, PA 19140, USA, Tel: 808-484-2288, Fax: 808484-1181, E-mail: CLee555294@aol.com

\begin{abstract}
Maxillary sinus membrane perforation or tear is the most common complication of the sinus grafting procedure. Repair of the sinus membrane is usually accomplished at the time of the sinus graft procedure and often results in uneventful postoperative complications. However, complications may still arise, especially with large sinus membrane perforations or complete tears that could lead to an infection of the maxillary sinus and other anatomic areas of the maxillofacial complex that could result in bone graft and dental implant failure. An alternative strategy and method to repair the Scheiderian membrane that results in a newly formed fibrotic sinus membrane is described that permits completion of dental implant treatment.
\end{abstract}

\section{Keywords}

Re-entry, Maxillary sinus, Infection, Sinusitis, Histology, Fibrotic Scheiderian membrane, Bioresorbable collagen membrane, Platelet rich plasma

\section{Introduction}

The maxillary sinus lift elevation procedure with bone graft augmentation is the most frequently performed surgical procedure to increase the vertical bone height of the posterior maxilla in preparation for dental implant placement. The procedure was first described by Tatum in 1976 [1-3] and later published by Boyne and James in 1980 [4]. Since the initial publication, the procedure has been modified by others and has a high clinical success rate of greater than 90\% [5-9]. However, this surgical procedure to correct the atrophic posterior maxilla is not without complications. Boyne [10] hypothesized that the size of the sinus membrane perforation may affect the success of the sinus bone graft augmentation procedure.

The most common intraoperative complication reported with grafting of the maxillary sinus is a perforation of the Schneiderian membrane (Figure 1) that is often managed at the time the perforation is observed [10-18]. The incidence of sinus membrane perforation reported in the literature ranges from 7 to $56 \%$ $[10,11,12,16]$. A meta-analysis by Jensen and Terheyden [19] revealed that infections occur as high as $4.7 \%$ with this surgical procedure. If the sinus infection is not identified and managed early in the course of treatment, such a complication can lead to the development of acute and chronic sinusitis, displacement of the graft material into the sinus cavity, loss of the graft material and oroantral communication [19-22]. Perforation of the sinus membrane may also effect homeostasis of the maxillary sinus and bacterial colonization into the maxillary sinus [12,16-18,20-22]. In more severe cases, the infection may progress to involve other anatomic structures of the maxillofacial region, such as the other paranasal sinuses, orbit and anterior and middle cranial fossae [24-26]. 


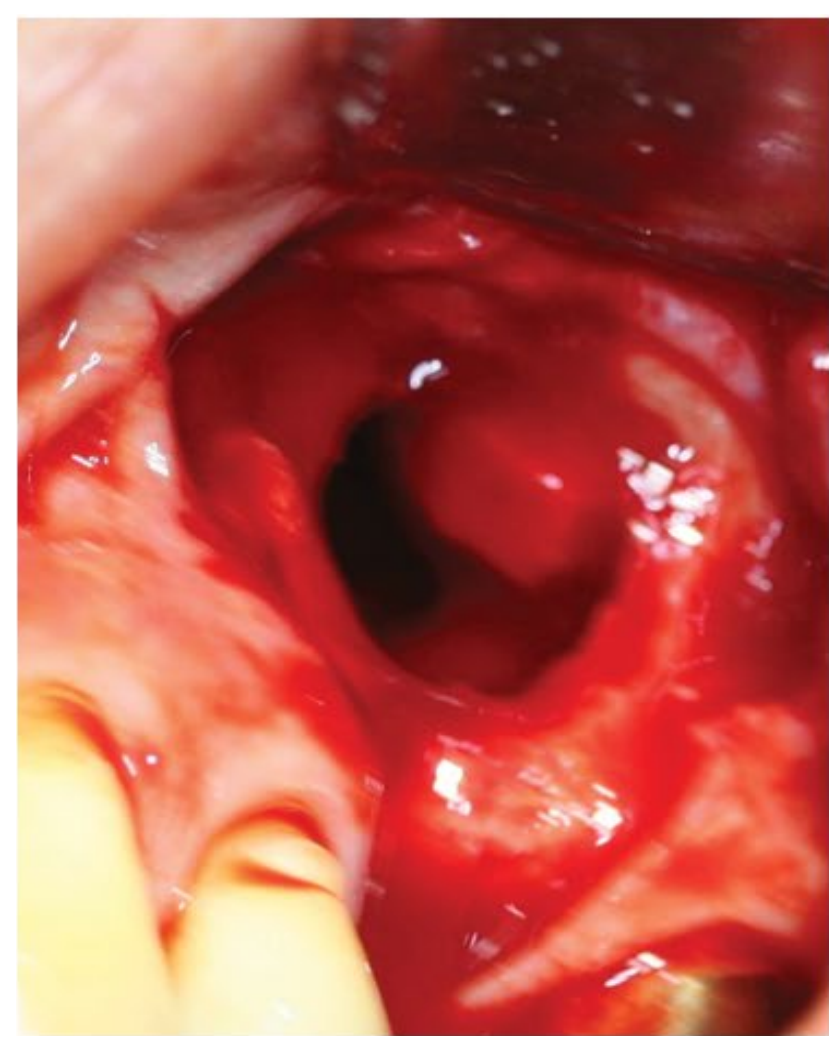

Figure 1: Intraoperative view of large perforation of the sinus membrane that occurred during the sinus lift elevation procedure.

The integrity of the Schneiderian membrane to prevent infection of the sinus cavity, loss of graft material and successful implant osseointegration cannot be under estimated. Several studies have shown a correlation between infection of the sinus and bone graft and implant failures with perforation of the sinus membrane at the time of sinus grafting [12,18,22-33]. A literature review regarding re-entry to manage a large perforation, or even complete sinus membrane tear associated with sinus augmentation procedures revealed only one article by Mardinger, et al. [33]. The goal of this article is to describe the clinical and histological changes of the buccal gingival tissues and the Schneiderian membrane that allow re-entry into the maxillary sinus to continue grafting of the maxillary sinus and dental implant placement. Such an alternative staged surgical strategy may avoid the postoperative complications associated with grafting the sinus cavity in the presence of a large perforation or complete tear of the sinus membrane.

\section{Histology of the Schneiderian Membrane of the Maxillary Sinus}

Like all sinuses, the maxillary sinus and nasal cavity are lined by a three-layered mucous membrane similar to that of the respiratory tract [34]. The sinus membrane varies in thickness from 0.3 to $0.8 \mathrm{~mm}$. It is continuous with the nasal epithelium, but thinner and not as vascular. The epithelial layer consists of pseudostratified ciliated columnar cells, basal cells and mucous secreting goblet cells. The subepithelial layer consists of

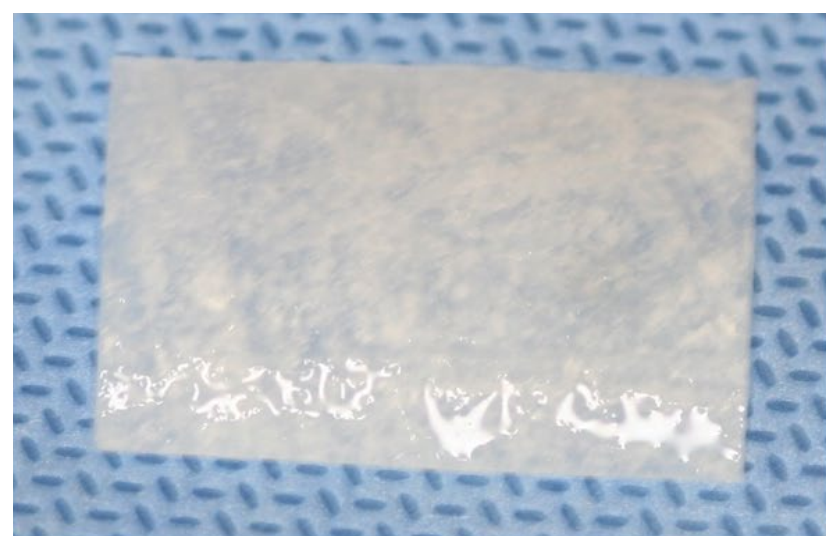

Figure 2A: Collagen membrane that will be trimmed and placed on the perforated sinus membrane.

collagen bundles and elastic fibers that are moderately vascularized. The periosteum is intimately attached to the subepithelial layer and is attached to the osseous walls of the sinus membrane.

\section{Management of the Large Perforated or Com- pletely Torn Schneiderian Membrane}

There are many methods and techniques that have been published regarding management of the sinus membrane perforation, as this is the most frequent complication with the sinus elevation procedure with bone grafting [10-33]. A critical objective of sinus membrane repair is to prevent extravasation of the graft material into the sinus cavity. Schneiderian membrane perforations can be repaired with use of a bioresorbable collagen membrane (Figure 2A) autologous fibrin glue, demineralized freeze-dried human lamellar bone sheet, oxidized regenerated cellulose, sutures and with platelet rich plasma $[9,11-15,17,18,23,30,31,35-37]$.

With large sinus perforations greater than $15 \mathrm{~mm}$, Pikos described the use of a slow bioresorbable collagen membrane and platelet rich plasma (Figure 2B) that acts as an adhesive to repair both partial and complete sinus membrane tears $[12,36]$. However, with large sinus membrane perforations, some authors recommend aborting the sinus augmentation procedure due to the higher percentage of implant failures associated with such large perforations $[6,7,9,16,29,38-40]$. An alternative strategy is to repair the large sinus membrane tear or perforation using a bioresorbable collagen membrane and platelet rich plasma, but without adding the bone graft material. Twelve weeks or later after the sinus membrane has healed, the maxillary sinus can be re-entered and the bone graft material added to the floor with or without simultaneous implant placement.

\section{Surgical Technique for Large and Complete Si- nus Membrane Perforations}

When the clinician encounters a large perforation or tear (Figure 1) during the sinus lift procedure or cannot control the membrane from tearing further, the clinician 


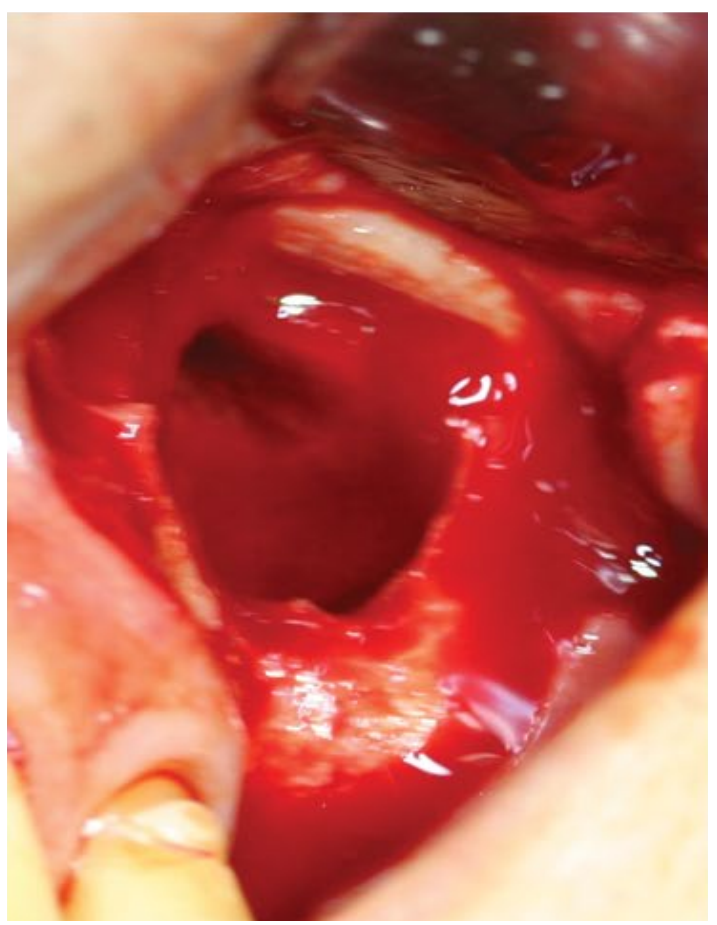

Figure 2B: Intraoperative view of collagen membrane and Platelet Rich Plasma (PRP) to repair and close off the perforated sinus membrane.

may elect to defer placing the graft material into the sinus cavity and focus on preparing the floor and walls to receive the graft material during the re-entry procedure that may occur 12 weeks or later. After the edges of the torn sinus membrane are elevated from the floor and walls of the sinus cavity and positioned medially, a bioresorbable collagen membrane can be directly placed over the sinus membrane. The clinician must make sure that the collagen membrane is not in contact with the floor and walls (anterior, medial and posterior) of the sinus cavity. Platelet Rich Plasma (PRP) is then sprayed on to the collagen membrane, sinus floor and walls (Figure 2B). Platelet rich plasma serves as a natural adhesive and barrier to the exposed sinus when applied to the bioresorbable collagen membrane and walls of the sinus cavity. More important, PRP has been shown to accelerate healing of soft and hard tissue as it is an autologous source of growth factors sequestered from the patient's own blood [41] that contain several different types of growth factors that can be applied to the surgical site to enhance wound healing. Such biologic growth mediators have been shown to stimulate bone regeneration and soft tissue healing by promoting angiogenesis and increased levels of vascular endothelial growth factor $[41,42]$.

\section{Reentry of the Maxillary Sinus and Histological Findings}

Twelve weeks or later after the sinus membrane has been repaired, re-entry into the maxillary sinus can be accomplished to add the graft material and place the implants. The newly formed fibrous buccal flap is continuous with the fibrotic Schneiderian membrane due to adhesions (Figure $3 \mathrm{~A}$ and Figure $3 \mathrm{~B}$ ) and may be difficult

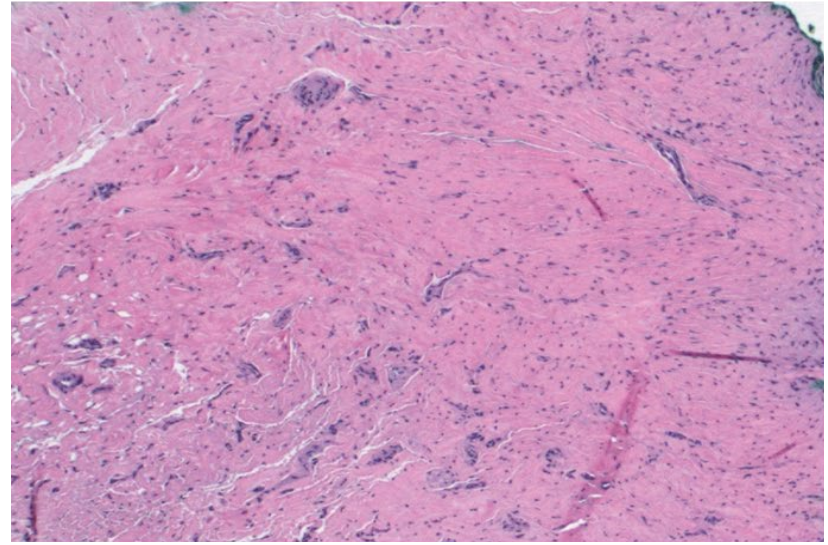

Figure 3A: Low power view of regenerated fibrotic Schneiderian membrane (H\&E stain. 40x).

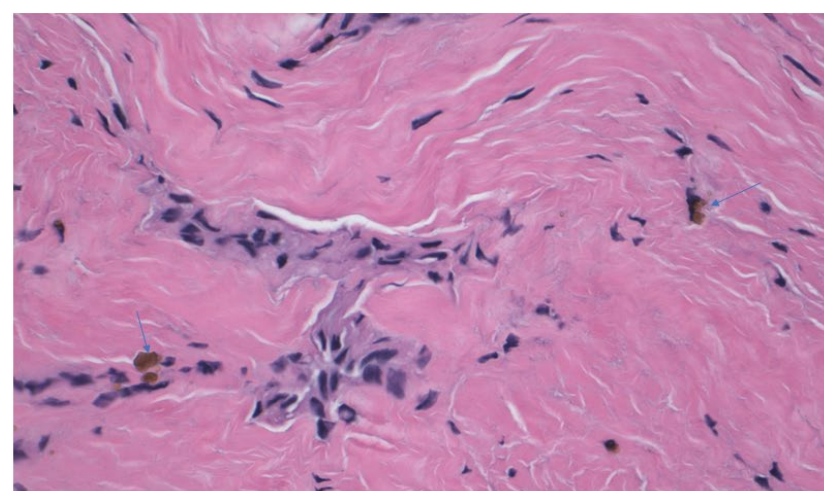

Figure 3B: High power view of regenerated Scheniderian membrane with capillary-sized vessels, fibroblasts and hemosiderin pigments (blue arrows) (H\&E stain. 200x).

to elevate the newly formed sinus membrane superiorly. Tearing of the sinus membrane when elevating in a medial direction is less likely due to the reparative process that results in a thick and fibrous sinus membrane (Figure 4A) that allows graft placement (Figure 4B).

\section{Discussion}

Many authors have reported on various techniques to repair the perforated and torn sinus membrane to complete the sinus elevation procedure, including grafting of the sinus [10-38]. Placing the graft material into the sinus cavity with a large perforation greater than $10.0 \mathrm{~mm}$ or more may result in an acute sinusitis that can progress to an infection of the sinus cavity, including the graft material. In such clinical scenarios, the graft material may need to be removed and the sinus cavity drained and irrigated to resolve the infection [2232]. Furthermore, studies by Proussaefs, et al. [18] and Khoury [29] reported higher implant failures with sinus membranes that were perforated. The study by Prossaefs and colleagues [18] also observed significant differences in formation of vital bone formation between non-perforated sinus membranes and perforated sinus membranes (33.6\% and $14.2 \%$ ) and implant survival (100\% versus $69.6 \%)$. Both authors hypothesize that the poor results associated with a repaired sinus membrane 


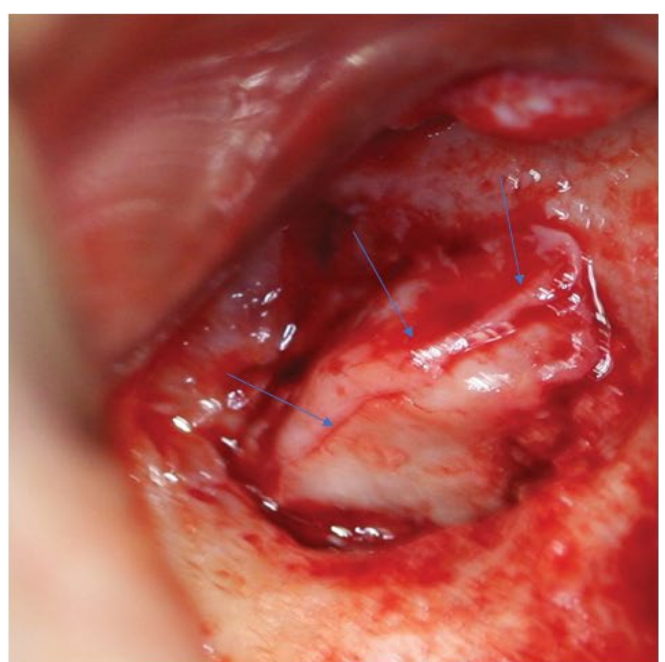

Figure 4A: Intraoperative view of thick fibrotic Schneiderian membrane (arrows) that develops 12 weeks after placement of the collagen membrane and platelet rich plasma on the perforated sinus membrane. Fibrotic membrane is elevated superiorly and medially to create a space to allow placement of graft material into the sinus cavity.

were due to colonization of bacteria into the bone graft material and bioresorbable collagen membrane used to repair the Schneiderian membrane and displacement of the graft material into the sinus Cavity $[18,29]$.

Based on a current literature review and to the best of the author's knowledge, there is only one published article that describes re-entry back into the maxillary sinus by Mardinger, et al. [33]. In their series of 17 patients that involved 21 reentered maxillary sinuses, they observed adhesions that limited mobility of the newly formed sinus membrane. Such adhesions and limited mobility still resulted in perforations or tears while attempting to elevate the sinus membrane in preparation for grafting the sinus. This was due to the reparative process of the sinus membrane that results in a nonflexible membrane that cannot fold on itself compared to a health sinus membrane that has the ability to do so. Such perforations had to be covered by bioresorbable collagen membranes to complete the sinus graft procedure.

Higher implant failure rates in the re-entered maxillary sinus were also observed [33]. Mardinger and colleagues hypothesize that the modified environment of the maxillary sinus may result in less formation of new bone and the ability of the dental implant to successfully osseointegrate [33]. In an animal study, Haas, et al. [43] observed an inflammatory reaction with a decrease in bone formation when the sinus membrane was perforated. In the author's surgical experience, this has not been observed with the staged re-entered grafted maxillary sinus. Staged re-entry of the maxillary sinus to complete the grafting procedure may prevent microbial pathogens from invading into and displacement of the graft material into the sinus cavity that is responsible for initiating an acute sinusitis and infection observed with immediately repaired sinus membrane perforations $[18,29]$.

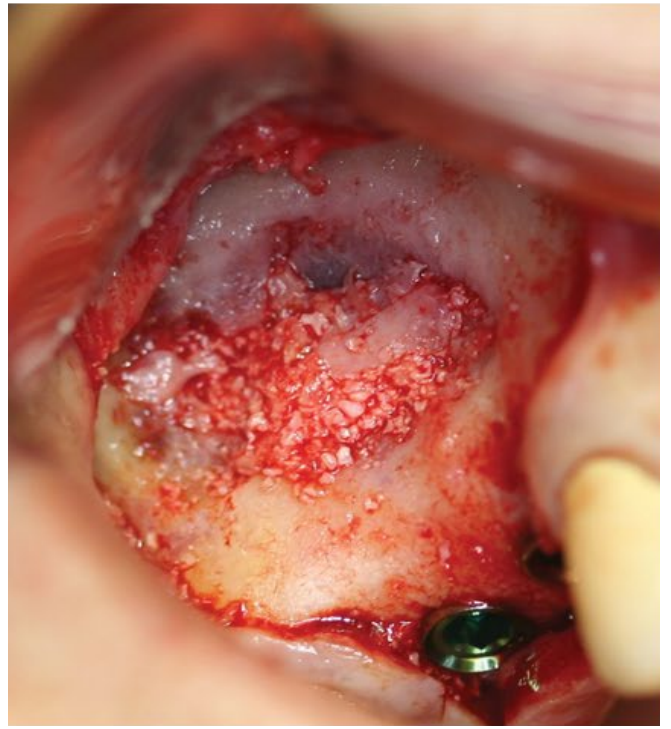

Figure 4B: Particulate allograft material mixed with PRP is placed into the sinus cavity.

\section{Conclusion}

This article describes an alternative surgical strategy and technique to assist the clinician in management of a large perforation or complete tear of the sinus membrane that avoids the postoperative complications associated with this surgical procedure. Staged re-entry of the maxillary sinus to graft the sinus cavity is predictable and increases graft material and implant survival.

\section{Disclosure}

The author declares no potential conflicts of interest with respect to financial funding, research, authorship and publication.

\section{References}

1. Tatum $\mathrm{OH}$ (1977) Maxillary sinus grafting for endosseous implants.

2. Tatum H Jr (1986) Maxillary and sinus implant reconstructions. Dent Clin North Am 30: 207-229.

3. Tatum OH Jr, Lebowitz MS, Tatum CA, Borgner RA (1993) Sinus augmentation. Rationale, development, long-term results. N Y State Dent J 59: 43-48.

4. Boyne PJ, James RA (1980) Grafting of the maxillary sinus floor with autogenous marrow and bone. J Oral Surg 38: 613-616.

5. Smiler DG, Holmes RE (1987) Sinus lift procedure using porous hydroxyapatite: A preliminary clinical report. J Oral Implantology 13: 239-253.

6. Wood RM, Moore DL (1988) Grafting of the maxillary sinus with intraorally harvested autogenous bone prior to implant placement. Int J Oral Maxillofac Implants 3: 209-214.

7. Kent JN, Block MS (1989) Simultaneous maxillary sinus floor bone grafting and placement of hydroxyapatite-coated implants. J Oral Maxillofac Surg 47: 238-242.

8. Jensen J, Sindet-Pedersen S (1991) Autogenous mandibular bone grafts and osseointegrated implants for reconstruction of the severely atrophied maxilla: A preliminary report. J Oral Maxillofac Implants 49: 1277-1287. 
9. Lundgren S, Moy P, Johansson C, Nilsson H (1996) Augmentation of the maxillary sinus floor with particulated mandible: A histologic and histomorphometric study. Int J Oral Maxillofac Implants 11: 760-769.

10. Boyne PJ (1993) Analysis of performance of root-form endosseous implants placed in the maxillary sinus. J Longterm Effects Med Implants 3: 143-159.

11. Barone A, Santini S, Sbordone L, Crespi R, Covani U (2006) A clinical study of the outcomes and complications associated with maxillary sinus augmentation. Int $\mathrm{J}$ Oral Maxillofac Implants 21: 81-88.

12. Pikos MA (2008) Maxillary sinus membrane repair: Update on technique for large and complete perforations. Implant Dent 17: 24-30.

13. Choi BH, Zhu SJ, Jung JH, Lee SH, Huh JY (2006) The use of autologous fibrin glue for closing sinus membrane perforations during sinus lifts. Oral Surg Oral Med Oral Pathol Oral Radiol Endod 191: 150-155.

14. Testori T, Wallace SS, Del Fabbro M, Taschieri S, Trisi P, et al. (2008) Repair of large sinus membrane perforations using stabilized collagen barrier membranes: surgical techniques with histologic and radiographic evidence of success. Int J Periodontics Restorative Dent 28: 9-17.

15. Sullivan SM, Bulard RA, Meaders R, Patterson MK (1997) The use of fibrin adhesive in sinus lift procedures. Oral Surg Oral Med Oral Pathol Oral Radiol Endod 84: 616-619.

16. Aimetti M, Romagnoli R, Ricci G, Massei G (2001) Maxillary sinus elevation: the effect of macrolacerations and microlacerations of the sinus membrane as determined by endoscopy. Int J Periodontics Restorative Dent 21: 581-589.

17. Proussaefs P, Lozada J, Kim J (2003) Effects of sealing the perforated sinus membrane with a resorbable collagen membrane: a pilot study in humans. J Oral Implantol 29: 235-241.

18. Proussaefs P, Lozada J, Kim J, Rohrer MD (2004) Repair of the perforated sinus membrane with a resorbable collagen membrane: a human study. Int $\mathrm{J}$ Oral Maxillofac Implants 19: 413-420.

19. Jensen SS, Terheyden H (2009) Bone augmentation procedures in localized defects in the alveolar ridge: clinical results with different bone grafts and bone-substitute materials. Int J Oral Maxillofac Implants 24: 218-236.

20. Chandra RK, Pearlman A, Conley DB, Kern RC, Chang D (2010) Significance of osteomeatal complex obstruction. J Otolaryngol Head Neck Surg 39: 171-174.

21. Timmenga NM, Raghoebar GM, Boering G (1997) Maxillary sinus function after sinus lifts for the insertion of dental implants. J Oral Maxillofac Surg 55: 936-939.

22. Schwarz L, Schiebel V, Hof M, Ulm C, Watzek G, et al (2015) Risk factors of membrane perforation and postoperative complications in sinus floor elevation surgery: review of 407 augmentation procedures. J Oral Maxillofac Surg 73 : $1275-1282$.

23. Katranji A, Fotek $P$, Wang $H L$ (2008) Sinus augmentation complications: etiology and treatment. Implant Dentistry 17: 339-349.

24. Quiney RE, Brimble E, Hodge M (1990) Maxillary sinusitis from dental osseointegrated implants. J Laryngol Otol 104: 333-334.

25. Timmenga NM, Raghoebar GM, van Weissenbruch $R$, Vissink A (2001) Maxillary sinusitis after augmentation of the maxillary sinus floor: a report of 2 cases. J Oral Maxillofac Surg 59: 200-204.
26. Alkan A, Celebi N, Bas B (2008) Acute maxillary sinusitis associated with internal sinus lifting: report of a case. Eur $\mathrm{J}$ Dent 2: 69-72.

27. Schwartz-Arad D, Herzberg R, Doley E (2004) The prevalence of surgical complications of the sinus graft procedure and their impact on implant survival. J Periodontol 75: 511-517.

28. Anavi Y, Allon DM, Avishai G, Calderon S (2008) Complications of maxillary sinus augmentations in a selective series of patients. Oral Surg Oral Med Oral Pathol Oral Radiol Endod 106: 34-39.

29. Khoury F (1999) Augmentation of the sinus floor with mandibular bone block and simultaneous implantation: a 6-year clinical investigation. Int J Oral Maxillofac Implants 14: 557-564.

30. Shlomi B, Horowitz I, Kahn A, Dobriyan A, Chaushu G (2004) The effect of sinus membrane perforation and repair with Lambone on the outcome of the maxillary sinus floor augmentation: A radiographic assessment. Int $\mathrm{J}$ Oral Maxillofac Implants 19: 559-565.

31. Hernandez-Alfaro F, Torradeflot MM, Marti C (2008) Prevalence and management of Schneiderian membrane perforations during sinus lift procedures. Clin Oral Implants Res 19: 91-95.

32. Nolan PJ, Freeman K, Kraut RA (2014) Correlation between Schneiderian membrane perforation and sinus lift graft outcome: a retrospective evaluation of 359 augmented sinus. J Oral Maxillofac Surg 72: 47-52.

33. Mardinger $O$, Moses $O$, Chaushu G, Manor $Y$, Tulchinsky $Z$, et al. (2010) Challenges associated with reentry maxillary sinus augmentation. Oral Surg Oral Med Oral Pathol Oral Radiol Endod 110: 287-291.

34. van den Bergh JP, ten Bruggenkate CM, Disch FJ, Tuinzing DB (2000) Anatomical aspects of sinus floor elevations. Clin Oral Implants Res 11: 256-265.

35. Simunek A, Kopeck D, Carney M (2005) The use of oxidized regenerated cellulose (surgical) in closing Schneiderian membrane tears during the sinus lift procedure. West Indian Med J 54: 398-399.

36. Pikos MA (1999) Maxillary sinus membrane repair: report of a technique for large perforations. Implant Dent 8: 29-34.

37. Oh E, Kraut RA (2011) Effect of sinus membrane perforation on dental implant integration: a retrospective study on 128 patients. Implant Dent 20: 13-19.

38. Becker ST, Terheyden H, Steinriede A, Behrens E, Springer I, et al. (2008) Prospective observation of 41 perforations of the Schneiderian membrane during sinus floor elevation. Clin Oral Implants Res 19: 1285-1289.

39. Fugazzotto P, Vlassis J (2003) A simplified classification and repair system for sinus membrane perforations. J Periodontol 74: 1534-1541.

40. Biglioli F, Pedrazzoli M, Colletti G (2010) Repair of a perforated sinus membrane with a palatal fibromucosal graft: a case report. Minerva Stomatol 59: 299-302.

41. Marx RE, Carlson ER, Eichstaedt RM, Schimmele SR, Strauss JE, et al. (1998) Platelet rich plasma: growth factor enhancement for bone grafts. Oral Surg Oral Med Oral Pathol Oral Radiol Endod 85: 638-646.

42. Marx RE (2004) Platelet-rich plasma: evidence to support its use. J Oral Maxillofac Surg 62: 489-496.

43. Haas R, Baron M, Donath K, Zechner W, Watzek G (2002) Porous hydroxyapatite for grafting the maxillary sinus: a comparative histomorphometric study in sheep. Int J Oral Maxillofac Implants 17: 337-346. 